\title{
A novel corona core-shell nanoparticle for enhanced intracellular drug delivery
}

\author{
JIANWEI JIANG ${ }^{1,2}$, XIAOMING ZHONG ${ }^{1}$, HONGYAN ZHANG ${ }^{2}$ and CHUNLEI WANG ${ }^{2}$ \\ ${ }^{1}$ College of Pharmaceutical Science, Zhejiang Chinese Medical University, Hangzhou, Zhejiang 310053; \\ ${ }^{2}$ Department of Pharmacy, Zhejiang Cancer Hospital, Hangzhou, Zhejiang 310022, P.R. China
}

Received January 6, 2019; Accepted December 23, 2019

DOI: $10.3892 / \mathrm{mmr} .2020 .10998$

\begin{abstract}
Drugs, including small molecule anticancer drugs, have to be delivered and released into the cytoplasm or nucleus in order to elicit a therapeutic effect. In the present study, a novel corona core-shell nanoparticle was proposed for enhanced cellular uptake and light-responsive intracellular release. Light-responsive core-shell nanoparticles, i.e., gold nanoparticles-coated liposomes (CS), were prepared and further modified with hyaluronic acid (HA) (CCS). HA modification enhances the endocytosis of the nanoparticles by HA receptor-expressing cells, while the plasmonic properties of gold nanoparticles enable their light-triggered drug release. The results demonstrated that the uptake of CCS in HA receptor-expressing MDA-MB-231R cells was significantly enhanced compared with unmodified CS. Nanosecond pulsed laser irradiation $\left(700 \mathrm{~nm} ; 100 \mathrm{~mJ} / \mathrm{cm}^{2}\right.$; 5 pulses) rapidly triggered the intracellular release of a fluorescent dye, 6-carboxyfluorescein (6-CF), from CCS and CS trapped in endolysosomes. The released $6-\mathrm{CF}$ was evenly distributed throughout the entire cytosol and nucleus. Finally, the cytotoxicity of doxorubicin towards MDA-MB-231 cells was significantly increased by CCS delivery upon pulsed laser irradiation. In conclusion, with enhanced cellular uptake and light-triggered intracellular release, CCS significantly enhanced the therapeutic effects of doxorubicin, and may serve as a promising avenue for intracellular drug delivery.
\end{abstract}

Correspondence to: Dr Xiaoming Zhong, College of Pharmaceutical Science, Zhejiang Chinese Medical University, 548 Binwen Road, Hangzhou, Zhejiang 310053, P.R. China

E-mail: zhongxm_1@sina.com

Dr Chunlei Wang, Department of Pharmacy, Zhejiang Cancer Hospital, 38 Guangji Road, Hangzhou, Zhejiang 310022, P.R. China E-mail: chenfc68@163.com

Key words: intracellular delivery, light responsive release, corona core-shell nanoparticle, hyaluronic acid, controlled release, cancer therapy

\section{Introduction}

Despite numerous advances in biotechnology, cancer is still the leading cause of death worldwide, responsible for $\sim 1 / 4$ of mortality in the USA (1). Several nanoscale drug delivery systems, including polymeric nanoparticles, liposomes and nanogels, have been developed for modern cancer therapy $(1,2)$. Compared with other traditional approaches, such as injection of free drug solution or drug loaded microparticles, these nanoscale drug formulations have demonstrated several advantages, including decreased adverse effects, improved pharmacological profiles and drug tolerance and prolonged circulation (3). Despite their merits, the majority of nanoscale drug delivery systems exhibit inefficient drug release and cellular uptake, which largely compromises the drug treatment benefits in the clinic (3). To elicit their therapeutic effects, many drugs, including small molecule drugs, proteins and small interfering RNAs, have to be delivered and released into specific cellular compartments, such as the cytoplasm of pathological cells. Therefore, to ensure sufficient drug levels in the pathological cells, drug delivery systems have to enter the target cells and efficiently release the drug molecules into the targeted cell compartment (4). In the past decade, a number of studies have investigated methods of enhancing cellular uptake and achieving rapid intracellular release; however, only a few approaches were able to achieve both of these effects (5-7).

Multifunctional hybrid drug delivery systems, including polymer or nanoparticle modified liposomes, exhibit the advantages of two or more delivery systems and have become one of the most popular delivery systems (8-10). A previous study revealed that light-responsive core shell nanoparticles, i.e., gold nanoparticle-coated liposomes, achieved rapid intracellular drug release in MDA-MB-231-R cancer cells (11). Under short pulsed laser irradiation $(532 \mathrm{~nm}$; $6 \mathrm{~ns})$, highly localized heat was generated owing to the surface plasmon resonance of the gold nanoparticle shell, and the encapsulated cargo was rapidly released due to increased membrane permeability of the liposomal core. Although the developed system was effective for intracellular drug release and enhancing drug therapeutic efficacy, the trigger used was green light (532 nm), which exhibits poor tissue penetration and photo-damage to biological tissues and cells. The deep tissue penetration and reduced photo-damage of near-infrared light (NIR) make it a particularly attractive option for biomolecule release (12-16). 
Therefore, further optimization of the light-triggered release system by switching the trigger from green light to NIR light is required.

In the present study, to further enhance the cellular uptake and achieve a high intracellular drug concentration, the core shell nanoparticles described by Jiang et al (11) were further modified by hyaluronic acid (HA), forming corona core-shell nanoparticles. HA, a naturally occurring high-molecular weight glycosaminoglycan, is a major component of the extracellular matrix (17). As a natural excipient, HA has several advantages, such as preventing protein absorption due to its hydrophilic and polyanionic characteristics in a physiological environment (18). Notably, HA has demonstrated high efficiency in targeting to tissues with HA receptors, such as classification determinant 44 (CD44) and hyaluronan receptor for endocytosis (HARE) (19). A number of cancer cells upregulate the expression of the HA receptor CD44, which has been proposed as a marker of breast cancer stem cells (20). On the basis of these findings, the present study developed an HA-modified hybrid nanoparticle (CCS) as an alternative to the unmodified CS to achieve higher intracellular drug concentration upon light activation and to further enhance therapeutic effects.

In the present study, CCS were developed and characterized by transmission electron microscopy (TEM), dynamic laser light scattering (DLS) and ultraviolet-visible (UV-Vis) spectroscopy. The results revealed that HA modification enhanced cellular uptake in MDA-MB-231 cells 1.9-fold. Pulsed laser irradiation rapidly released encapsulated 6-carboxyfluorescein (6-CF) from CCS and CS trapped in endosomes and lysosomes into the cytosol and nucleus. Furthermore, the cytotoxicity of doxorubicin was significantly enhanced by CCS delivery upon laser irradiation. The results showed that HA-modified light responsive nanoparticles were able to achieve high intracellular drug concentration by enhancing cellular uptake and had rapid intracellular drug release upon light activation, suggesting that this approach may be a promising intracellular drug delivery system.

\section{Materials and methods}

Materials. 1,2-Dipalmitoyl-sn-glycero-3-phosphocholine (DPPC) and cholesterol were purchased from Avanti Polar Lipids; Merck KGaA. Sodium hyaluronate, cystamine hydrochloride, hyaluronidase (type I-S), NaBH3CN, gold (III) chloride trihydrate and 6-CF were purchased from Sigma-Aldrich; Merck KGaA. Ascorbic acid, borate buffer were obtained from Thermo Fisher Scientific, Inc. DMEM and fetal bovine serum were purchased from Gibco; Thermo Fisher Scientific, Inc. 4',6-Diamidino-2-phenylindole (DAPI) and LysoTracker Red DND-99 were purchased from Thermo Fisher Scientific, Inc. Doxorubicin sodium salt was purchased from Alfa Aesar. All other reagents were of analytical grade and used without further purification.

Synthesis of thiol end-functionalized HA. A low-molecular weight HA was obtained via digestion of HA with hyaluronidase (type I-S) following a previously reported method (21). Briefly, hyaluronidase was added into the HA solution $(10 \mathrm{mg} / \mathrm{ml} ; \mathrm{pH} 7.0)$ at a ratio of 100 units of hyaluronidase per mg of HA. The mixture was incubated at $37^{\circ} \mathrm{C}$ for $20 \mathrm{~h}$, followed by boiling $\left(100^{\circ} \mathrm{C}\right)$ for $10 \mathrm{~min}$ to inactivate the hyaluronidase. An Amicon ultrafiltration kit [molecular weight cut-off (MWCO); 1,000 Da; Amicon Corporation] was used to remove aggregated hyaluronidase and high molecular weight HA. The filtrated solution was then dialyzed (MWCO, $1,000 \mathrm{Da}$ ) for $24 \mathrm{~h}$ under $25^{\circ} \mathrm{C}$ to remove 1,2 or 3 mer HA. Dry oligo-HA was obtained after the lyophilization of the HA solution following dialysis. For lypholiziation, HA solutions were placed into $25 \mathrm{ml}$ glass vials and kept at $-80^{\circ} \mathrm{C}$ for $2 \mathrm{~h}$, followed by freeze-drying (Lio-Labor ${ }^{\circledR}$; Telstar) for $48 \mathrm{~h}$ to reach a freezing temperature $\left(-45^{\circ} \mathrm{C}\right)$, a sublimation temperature (from $\left.-45-25^{\circ} \mathrm{C}\right)$ and a sublimation pressure $\left(4.54 \times 10^{-4}\right.$ atmosphere). For thiol modification at the reducing end, $50 \mathrm{mg}$ of oligo-HA and $60 \mathrm{mg}$ of cystamine hydrochloride were dissolved in borate buffer ( $\mathrm{pH} 8.5 ; 0.4 \mathrm{M} \mathrm{NaCl}$ ). Sodium borohydride ( $\mathrm{NaBH} 3 \mathrm{CN})$ was added to the reaction mixture at a final concentration of $200 \mathrm{mM}$ and incubated at $40^{\circ} \mathrm{C}$ for 5 days. Free thiol groups were obtained by incubation of the resulting mixture with $100 \mathrm{mM}$ dithiothreitol (DTT) at $25^{\circ} \mathrm{C}$ for $12 \mathrm{~h}$. The mixture was further dialyzed (MWCO: 1,000 Da) for $24 \mathrm{~h}$ at $25^{\circ} \mathrm{C}$ to remove unreacted chemicals and was subsequently lyophilized using the same protocol as HA lyophilization.

Preparation of CCS and CS. Gold nanoparticle coated liposomes (CS) were prepared following a previously reported method (8). Briefly, liposomes were prepared with DPPC and cholesterol at a molar ratio of 70:30. Lipid powders were dissolved in chloroform and dried under nitrogen stream, and then placed in a vacuum overnight to completely remove chloroform. For the encapsulation of doxorubicin, the lipid film was hydrated with $300 \mathrm{mM}$ ammonium sulfate $(\mathrm{pH}=7.5)$, followed by extrusion for 21 times through a $200 \mathrm{~nm}$ polycarbonate membrane using an Avanti mini-extruder (Sigma-Aldrich; Merck KGaA). Empty liposomes were then passed through the S1000 column (GE Healthcare) pre-equilibrated with isotonic N-2-hydroxyethylpiperazine-N-2-ethanesulfonic acid (HEPES) buffer (140 mM NaCl; $10 \mathrm{mM}$ HEPES; $\mathrm{pH}=7.4$ ) to remove the extra ammonium sulfate solution. Subsequently, doxorubicin hydrochloride was added to the liposome suspension to achieve a drug to lipid ratio of $1: 3(\mathrm{~mol} / \mathrm{mol})$. The loading process was carried out at $37^{\circ} \mathrm{C}$ for $2 \mathrm{~h}$. For the encapsulation of 6-CF, the drug-lipid film was hydrated with 6-CF solution $(0.1$ or $100 \mathrm{mM})$ at $55^{\circ} \mathrm{C}$ for $1 \mathrm{~h}$. The free doxorubicin or 6-CF was removed by size extrusion chromatography eluted with HEPES buffer with a flow rate of $1 \mathrm{ml} / \mathrm{min}$. The samples were detected at $280 \mathrm{~nm}$.

For gold coating, liposomes were diluted to $1 \mathrm{mM}$ using HEPES buffer. Gold chloride solution at a concentration of $20 \mathrm{mM}$ was added and mixed with liposomes, followed by addition of ascorbic acid solution (40 mM). Following reduction, gold liposomes were dialyzed against HEPES buffer for $24 \mathrm{~h}$ at $4^{\circ} \mathrm{C}$ to remove unreacted gold chloride and ascorbic acid. The thiol end-functionalized HA (HA-SH) was then immobilized onto the surface of CS via gold-thiol chemistry (21). Briefly, HA-SH was incubated with CS solutions at room temperature for $2 \mathrm{~h}$, and then the unreacted $\mathrm{HA}-\mathrm{SH}$ was removed by dialysis using a dialysis tubing (MWCO, 12,000 Da; Sigma-Aldrich; Merck KGaA). The resulting CCS samples were stored at $4^{\circ} \mathrm{C}$ until further use. 
The hydrodynamic radius and polydispersity of liposomes, CS and CCS were determined by diffraction light scattering (DLS) method using a Zetasizer (Nano ZS; Malvern Panalytical). The UV-Vis spectra in the range of 400-1,100 nm were recorded using a spectrometer. The morphology of the CCS samples was observed by TEM operating at a voltage of $200 \mathrm{kV}$. CCS samples were diluted with HEPES buffer and placed onto a carbon film coated copper grid. The samples were air-dried for $2 \mathrm{~h}$ at room temperature and subsequently imaged using a $125 \mathrm{~K}$ magnification.

Cytotoxicity and cellular uptake of CS and CCS. The cytotoxicity of CS and CCS on MDA-MB-231 cells was evaluated using the XTT method following a previously reported method (22). MDA-MB-231 cells from the American Type Culture Collection were cultured in a 96-well plate (1x10 4 cells/well) using DMEM with $10 \%$ FBS and incubated for $24 \mathrm{~h}$ at $37^{\circ} \mathrm{C}$. The cells were treated with blank CS and CCS $(0.1,0.5,1.0 \mathrm{mg} / \mathrm{ml})$ for $4 \mathrm{~h}$ at $37^{\circ} \mathrm{C}$. After aspirating the treatment media, $100 \mu \mathrm{l}$ of DMEM and $25 \mu \mathrm{l}$ of XTT solution were added to the cells. The control cells were treated with medium alone without $\mathrm{CS}$ or CCS. After a 4-h incubation at $37^{\circ} \mathrm{C}$, the absorbance was measured at a wavelength of $450 \mathrm{~nm}$ using a Multiskan GO microplate reader (Thermo Fisher Scientific, Inc.). Cell viability was calculated as a percentage of control cells.

Flow cytometry was used to quantify the endocytosed nanoparticles and the effects of free HA on the endocytosis efficiency of nanoparticles. MDA-MB-231 cells were seeded into a 24-well plate at a density of $2 \times 10^{5}$ cells $/ \mathrm{ml}$ and cells in the preconfluent state were used for uptake studies. The cells were incubated with $6-\mathrm{CF}(0.1 \mathrm{mM})$ encapsulated $\mathrm{CS}$ and $\mathrm{CCS}$ for $4 \mathrm{~h}$ at $37^{\circ} \mathrm{C}$, washed three times with ice-cold PBS, and subsequently harvested and analyzed using a ZE5 cell analyzer (Everest software 2.2; Bio-Rad Laboratories, Inc.). Cells incubated with medium alone without any nanoparticles were used as the negative control. A total of 10,000 cells were analyzed in each group. To investigate whether the cellular uptake of CCS was mediated by the HA receptors, the cells were pretreated with free HA $(10 \mathrm{mg} / \mathrm{ml}$, hydrated overnight in serum-free DMEM) for $1 \mathrm{~h}$ at $37^{\circ} \mathrm{C}$ before the addition of nanoparticles.

To investigate the intracellular distribution of CS and CCS, cells with endocytosed nanoparticles were observed by confocallaser scanning microscopy (CLSM; FV1000; Olympus Corporation). Briefly, MDA-MB-231 cells were seeded in $25-\mathrm{mm}$ glass bottom dishes at a density of $1 \times 10^{5}$ cells/plate and cultured at $37^{\circ} \mathrm{C}$ for $24 \mathrm{~h}$. The DMEM was then replaced with $1 \mathrm{ml}$ of medium containing CCS or CS and incubated at $37^{\circ} \mathrm{C}$ for $4 \mathrm{~h}$. After the nanoparticle containing medium was removed, late endosomes and lysosomes were stained with LysoTracker Red DND-99 at $37^{\circ} \mathrm{C}$ for $30 \mathrm{~min}$. Cells were then supplied with fresh medium and immediately observed under a confocal laser microscope using x100 magnification.

Light-triggered intracellular release. To monitor pulsed laser-triggered release, CS and CCS were encapsulated with $100 \mathrm{mM}$ 6-CF, a concentration at which its fluorescence is self-quenched (16). The cell nuclei of MDA-MB-231 cells cultured in $25-\mathrm{mm}$ glass bottom dishes at a density of
Table I. Hydrodynamic diameter and polydispersity of uncoated liposomes, CS and CCS.

\begin{tabular}{lcc}
\hline Sample & Diameter $(\mathrm{nm})$ & Polydispersity index \\
\hline Uncoated liposomes & $185 \pm 10$ & $0.051 \pm 0.012$ \\
CS & $210 \pm 18$ & $0.138 \pm 0.039$ \\
CCS & $227 \pm 21$ & $0.163 \pm 0.031$ \\
\hline
\end{tabular}

The data are presented as mean \pm SD for three independent experiments. Polydispersity indices were measured by Zetasizer. CCS, HA modified gold nanoparticles coated liposomes; CS, gold nanoparticles-coated liposomes.

$1 \times 10^{5}$ cells/plate were stained with $5 \mu \mathrm{g} / \mathrm{ml}$ Hoechst 33342 at $37^{\circ} \mathrm{C}$ for $5 \mathrm{~min}$, and then cells were treated with $6-\mathrm{CF}$ encapsulated CS and CCS $(100 \mathrm{mM}) 37^{\circ} \mathrm{C}$ for $4 \mathrm{~h}$. Cells were subsequently washed with $\mathrm{PBS}$ and supplied with fresh DMEM prior to laser irradiation $\left(700 \mathrm{~nm} ; 100 \mathrm{~mJ} / \mathrm{cm}^{2}\right.$; 5 pulses), and then immediately observed by CLSM using x100 magnification.

Cytotoxicity of doxorubicin-containing CS and CCS. The effects of CCS and pulsed laser irradiation on the cytotoxicity of doxorubicin on MDA-MB 231-R cells were investigated using the XTT method. MDA-MB 231-R cells were cultured overnight in 96-well plates at a density of $1 \times 10^{4} /$ well at $37^{\circ} \mathrm{C}$. The control group consisted of free doxorubicin-treated cells, and the treatment groups were as follows: i) Doxorubicin-encapsulated CS with or without laser irradiation; and ii) doxorubicin-encapsulated CCS with or without laser irradiation. The cells were washed with PBS and incubated with fresh DMEM that contained doxorubicin encapsulated in $\mathrm{CS}$ or CCS for $3 \mathrm{~h}$ at $37^{\circ} \mathrm{C}$. The cells were washed 3 times with PBS, and the laser-treated groups were irradiated with nanosecond (ns) laser pulse (700 nm; 5 pulses; $100 \mathrm{~mJ} / \mathrm{cm}^{2}$ ), followed by the addition of fresh DMEM and incubation for $24 \mathrm{~h}$ at $37^{\circ} \mathrm{C}$. Subsequently, the cell viability was determined by the XTT assay as previously mentioned. The drug concentration causing $50 \%$ inhibition $\left(\mathrm{IC}_{50}\right)$ was calculated.

Statistical analysis. All data are presented as the mean \pm standard deviation of three independent experiments. The unpaired Student's t-test was used to compare two groups. Three or more groups were analyzed using the one-way ANOVA followed by the Dunnett's post hoc. $\mathrm{P}<0.05$ was considered to indicate a statistically significant difference.

\section{Results}

Preparation and characterization of CCS. CCS nanoparticles were prepared using a three step method as shown in Fig. 1A. The CCS had a hydrodynamic size of $\sim 227 \mathrm{~nm}$, while uncoated liposomes and CS measured $\sim 185$ and $210 \mathrm{~nm}$, respectively (Table I). CCS is monodispersed with Polydispersity index of 0.163. TEM observations revealed that the CCS exhibited a typical coronal core shell structure as expected. Gold 
A

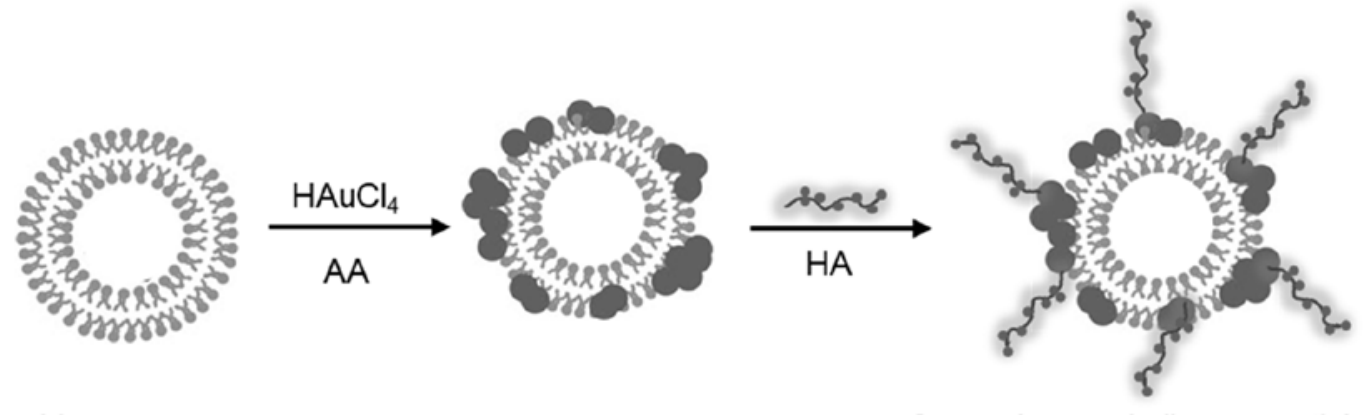

Liposomes

B

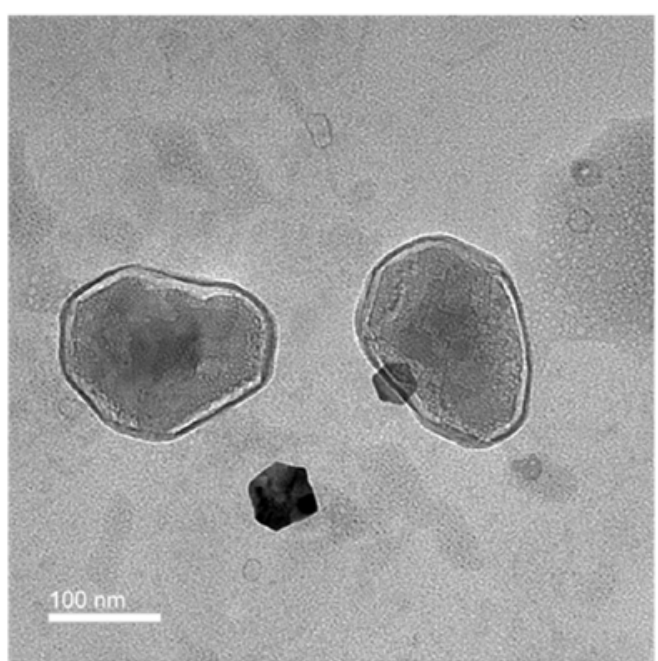

(CS)

C
Coronal core-shell nanoparticles

(CSC)

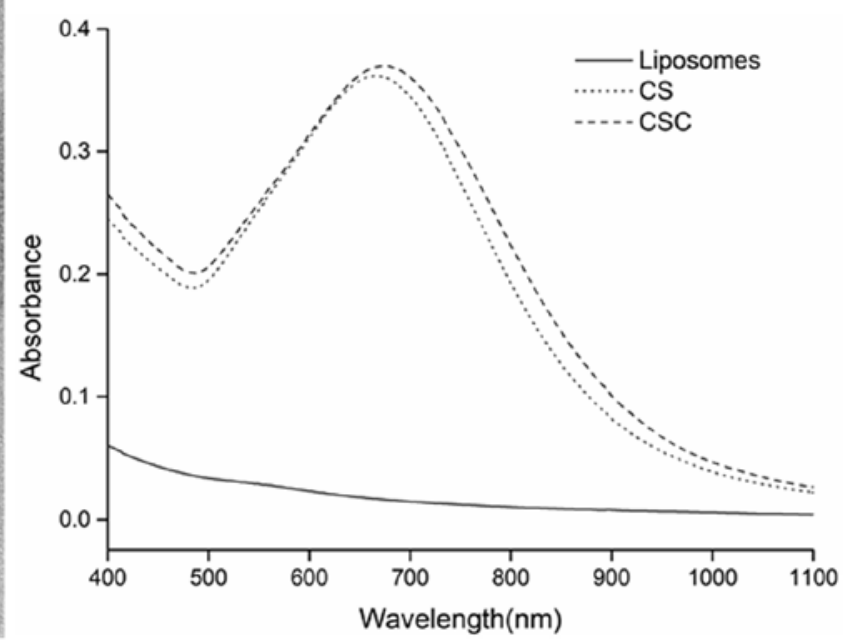

Figure 1. Preparation and characterization of CCS. (A) Schematic of the formation of CCS. (B) A typical transmission electron microscope image of CCS The average size of the particles was determined by dynamic laser light scattering. (C) Ultra violet-visible spectra of uncoated liposomes, CS and CCS. The two smaller black particles on the image represent salt precipitates. CCS, HA modified gold nanoparticles coated liposomes; CS, gold nanoparticles-coated liposomes; AA, ascorbic acid; $\mathrm{HA}$, hyaluronic acid; $\mathrm{HAuCl}_{4}$, gold (III) chloride trihydrate.

nanoparticles were distributed on the surface of the liposomal core, while HA formed a polymer corona on the outer surface (Fig. 1B). The TEM results suggested that HA had attached onto the gold nanoparticles on the surface. The UV-Vis spectra showed that CCS and CS exhibited an absorption peak at $\sim 673$ and $667 \mathrm{~nm}$, respectively, while uncoated liposomes did not show any resonance peak in the wavelength range of 400-1,100 nm (Fig. 1C). Gold liposomes presented high absorbance in the wavelength range $500-800 \mathrm{~nm}$, suggesting that gold liposomes could be activated by light in the range of 500-800 nm. CCS and CS were activated at $700 \mathrm{~nm}$ in subsequent experiments to ensure maximum absorbance in the NIR range.

Characterization of cellular uptake, cytotoxicity and intracellular distribution of CCS. The cytotoxicity of blank CS and CCS are shown in Fig. 2A. No significant cytotoxicity was observed for CS and CCS at the tested concentrations $(0.1,0.5$ and $1.0 \mathrm{mg} / \mathrm{ml})$. Quantitative analysis by flow cytometry revealed that HA modification significantly enhanced the cellular uptake of CS (Fig. 2B and C). The cellular uptake of CCS was $~ 1.9$-fold higher than CS in the absence of HA modification on the surface. To further verify whether the uptake of CCS was specific to HA receptors, competitive binding experiments were performed by pretreating MDA-MB-231 cells with excess free HA (200-400 kDa) before incubation. As shown in Figs. 2B and $\mathrm{C}$, ligand pretreatment did not change the cellular uptakes of CS, while the cellular uptake of CCS was significantly reduced. These results suggested that the free HA competed with CCS for receptor binding sites. Thus, cell surface HA receptors, mainly CD44 and HARE, may have mediated the cellular uptake process. Late endosomes and lysosomes were labeled with LysoTracker Red DND-99 to investigate the co-localization of 6-CF encapsulated CS and $\mathrm{CCS}$ with endolysosomes. As shown in Fig. 2D, following $4 \mathrm{~h}$ of incubation, the green fluorescence from 6-CF was highly co-localized with LysoTracker Red DND-99 (red fluorescence). The results indicated that endocytosed CCS and CS were largely trapped in endolysosomes and may have failed to release their cargo into the cytosol.

Pulse laser-triggered intracellular release. Upon activation by the ns pulsed laser, intracellular doxorubicin release was observed by CLSM imaging. The fluorescence of 6-CF at a high concentration was quenched due to its self-association. Release of 6-CF from liposomal particles and dilution by the surrounding medium de-quench 6-CF and increase its fluorescence intensity (16). The results demonstrated that upon short-pulsed laser irradiation $\left(700 \mathrm{~nm} ; 100 \mathrm{~mJ} / \mathrm{cm}^{2} ; 5\right.$ pulses $)$, 

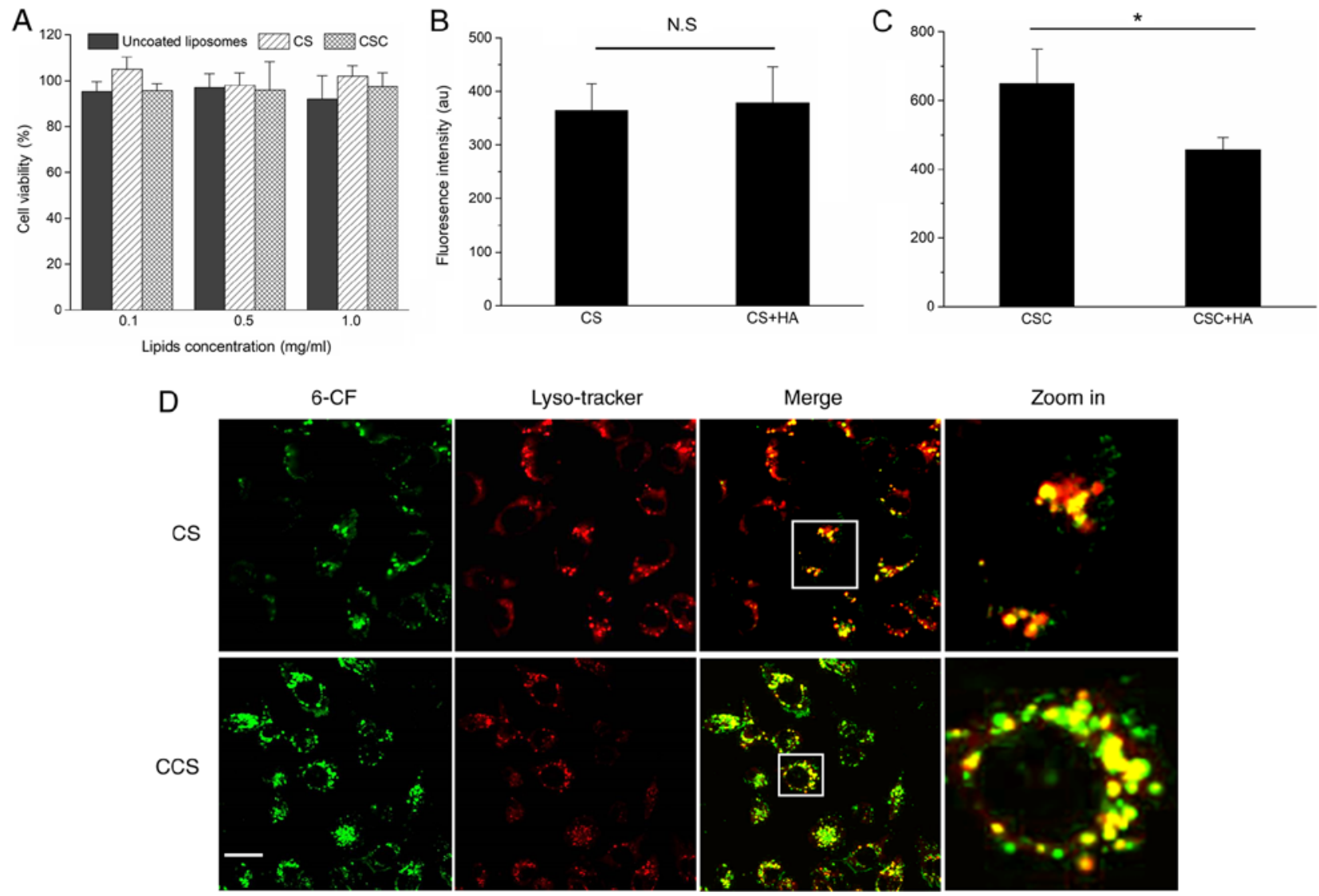

Figure 2. Cellular uptake and intracellular distribution of CS and CCS. (A) Cytotoxicity of blank CS and CCS with different concentrations. Quantitative analysis of the cellular uptake determined by flow cytometry of cells treated by CS (B) and CCS (C) with or without free HA (D) CLSM images of MDA-MB-231 cells treated with CS and CCS prior to laser irradiation suggested that the majority of CS and CCS were trapped in endolysosomes 6-CF is represented by the green fluorescence and LysoTracker Red DND-99 is represented by the red fluorescence. Scale bar, $20 \mu \mathrm{m}$. Magnification, x100. "P<0.05. CCS, HA modified gold nanoparticles coated liposomes; CS, gold nanoparticles-coated liposomes; 6-CF, 6-carboxyfluorescein; CLSM, confocal laser scanning microscopy; NS, not significant.

6-CF was rapidly released from CS and CCS trapped in endolysosomes and evenly distributed in the cytosol and nucleus (Fig. 3). This observation was in agreement with a previous study where gold nanoparticle liposomes were triggered at a different wavelength (532 $\mathrm{nm} ; 100 \mathrm{~mJ} / \mathrm{cm}^{2} ; 5$ pulses) (8). CCS exhibited a stronger green fluorescence intensity after laser activation, suggesting that increased cellular uptake by HA modification may have contributed to higher intracellular free drug concentration following activation.

Cytotoxicity of doxorubicin on MDA-MB-231 cells. To test the ability of CCS to enhance the therapeutic effects of doxorubicin, the cytotoxicity of doxorubicin-encapsulated CS and CCS with or without laser irradiation was measured by the XTT assay. The results demonstrated that laser irradiation markedly increased the cytotoxicity of doxorubicin in both CS and CCS (Fig. 4A). HA modification also enhanced the cytotoxicity of doxorubicin, as the $\mathrm{IC}_{50}$ of doxorubicin encapsulated in CCS decreased to $3.0 \mu \mathrm{M}$, while the $\mathrm{IC}_{50}$ of doxorubicin encapsulated in CS was $5.8 \mu \mathrm{M}$. Cytotoxicity enhancement was the most significant when doxorubicin was incorporated into CCS and activated by ns pulsed laser, and the $\mathrm{IC}_{50}$ was further decreased to $1.5 \mu \mathrm{M}$. Under the current experimental conditions, laser irradiation alone ( 5 pulses; $\left.100 \mathrm{~mJ} / \mathrm{cm}^{2}\right)$ did not result in significant cell death, as indicated in Fig. 4B.

\section{Discussion}

While several cancer drug delivery systems have been developed, limited curative effects in patients have been observed (2). Enhancing drug release from endocytosed drug carriers is of great significance since the majority of therapeutic agents have to be released into the cytosol or nucleus to elicit their therapeutic effects (3). A number of controlled drug release strategies have been reported in recent years, including diffusion-based and biologically-activated drug release systems (3,23-24). However, drug release based on these mechanisms is usually slow, cannot be precisely controlled and does not amplify the site selectivity of drug delivery (25). Strategies that enable fast drug release upon activation remain desirable to enhance intracellular cancer drug delivery. A previous study reported that burst release resulted in instant high intracellular drug concentration, which significantly enhanced doxorubicin cytotoxicity (11). In the present study, a light-triggered delivery system was optimized by using NIR laser pulses as the drug release trigger. The results demonstrated that similar to green light, ns NIR pulses triggered rapid intracellular drug release from CS and CCS trapped in endolysosomes.

In addition to increasing intracellular drug release, enhancing the cellular uptake of drug carriers is an important step to ensure a high intracellular concentration of free 


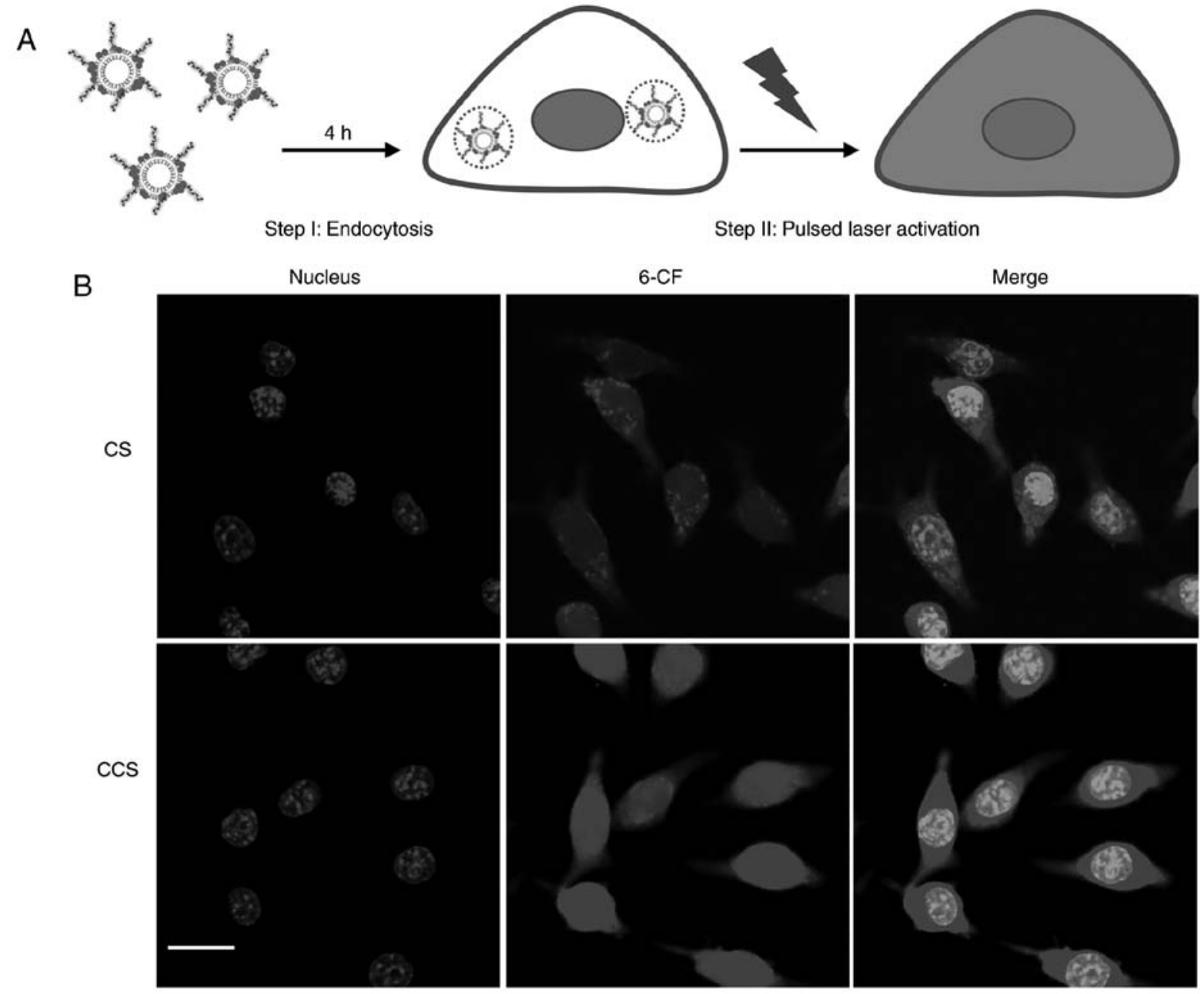

Figure 3. Intracellular light-triggered 6-CF release. (A) Schematic of the experimental procedure. (B) CLSM images of MDA-MB-231 cells following light irradiation indicating that 6-CF had been released and was evenly distributed throughout the whole cell. The cell nuclei were stained by Hoechst 33342 . Scale bar, $20 \mu \mathrm{m}$. Magnification, x100. 6-CF, 6-carboxyfluorescein; CLSM, confocal laser scanning microscopy.
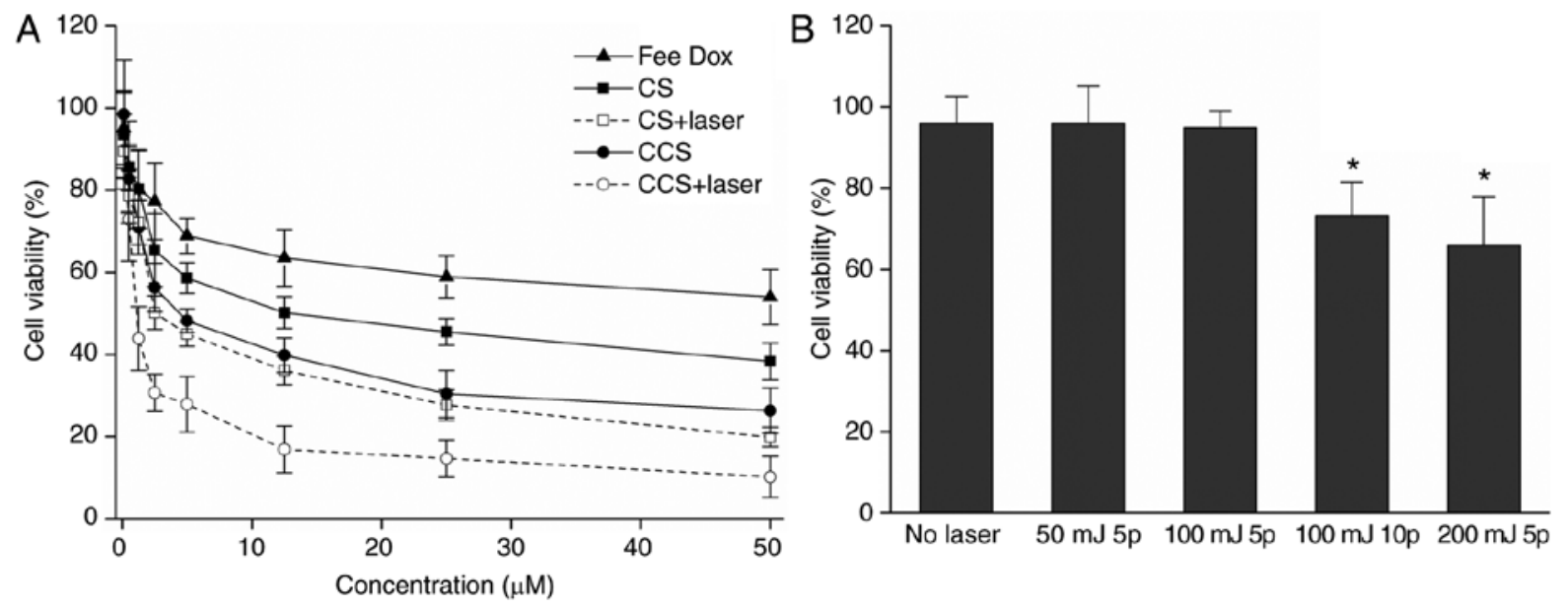

Figure 4. Cytotoxicity of doxorubicin. (A) Cell viability of MDA-MB-231 cells treated with free doxorubicin or doxorubicin encapsulated in CS or CCS with or without laser irradiation $\left(700 \mathrm{~nm} ; 5\right.$ pulses; $\left.100 \mathrm{~mJ} / \mathrm{cm}^{2}\right)$. ${ }^{*} \mathrm{P}<0.05 \mathrm{vs}$. free doxorubicin. (B) Cell viability of MDA-MB-231 cells treated with nanosecond pulsed laser with different pulse number or energy intensity. CCS, HA modified gold nanoparticles coated liposomes; CS, gold nanoparticles-coated liposomes.

drug (26). Therefore, in the present study, the light responsive nanoparticles, i.e., gold nanoparticle coated liposomes, were further modified by HA to increase the endocytosis efficiency and maximize the triggering effects. The results revealed that HA modification significantly increased the cellular uptake of nanoparticles, resulting in a greater intracellular drug 
concentration. HA modification alone significantly enhanced the cytotoxicity of doxorubicin as the $\mathrm{IC}_{50}$ in MDA-MB-231R cells decreased from 5.8 to $3.0 \mu \mathrm{M}$. When activated by short-pulsed laser $\left(700 \mathrm{~nm} ; 100 \mathrm{~mJ} / \mathrm{cm}^{2} ; 5\right.$ pulses $)$, the $\mathrm{IC}_{50}$ of doxorubicin was further decreased by $\sim 2$-fold. The results suggested that ligand-modified light responsive nanoparticles may be a promising drug delivery system for intracellular drug delivery by combining enhanced cellular uptake and rapid light-triggered intracellular release.

The advantages of using NIR as the light trigger include deep tissue penetration, minimum auto-fluorescence and tissue scattering as well as high biosafety (16). While CCS has demonstrated its potential as an intracellular drug delivery carrier at the cellular level, further in vivo studies are required to confirm its ability to enhance the therapeutic efficiency of drugs. Although NIR increases tissue penetration compared to green light (16), in vivo single site light delivery remains a challenge. Therefore, approaches that deliver a light trigger to target tissue are required prior to applying this technique in vivo.

In conclusion, the present study developed a dual functional CCS nanoparticle for intracellular drug delivery. Light responsive nanoparticles were further modified by HA, which enhanced the cellular uptake by 1.9-fold. In MDA-MB 231-R cancer cells, short pulsed laser $\left(700 \mathrm{~nm} ; 100 \mathrm{~mJ} / \mathrm{cm}^{2} ; 5\right.$ pulses $)$ liberated 6-CF from endocytosed CCS into the cytosol and nucleus. Furthermore, laser irradiation significantly increased the cytotoxicity of doxorubicin encapsulated in CCS in MDA-MB 231-R cells. The results suggested that a combination of active targeting and laser triggered on-demand release of chemotherapeutic agents may be a promising approach for intracellular drug delivery and may enhance therapeutic effects.

\section{Acknowledgements}

Not applicable.

\section{Funding}

This study was supported by the Zhejiang Province Medical and Health Technology Program (grant no. 2017K Y235).

\section{Availability of data and materials}

The datasets used and/or analyzed during the current study are available from the corresponding author on reasonable request.

\section{Authors' contributions}

JJ, XZ and CW designed the study. JJ and $\mathrm{HZ}$ performed the experiments. $\mathrm{XZ}$ and $\mathrm{CW}$ analyzed the results and wrote the manuscript.

\section{Ethics approval and consent to participate}

Not applicable.

\section{Patient consent for publication}

Not applicable.

\section{Competing interests}

The authors declare that they have no competing interests.

\section{References}

1. Dreaden EC, Austin LA, Mackey MA and El-Sayed MA: Size matters: Gold nanoparticles in targeted cancer drug delivery. Ther Deliv 3: 457-478, 2012

2. Lukianova-Hleb EY, Ren X, Sawant RR, Wu X, Torchilin VP and Lapotko DO: On-demand intracellular amplification of chemoradiation with cancer-specific plasmonic nanobubbles. Nat Med 20: 778-784, 2014.

3. Meng FH, Cheng R, Deng C and Zhong ZY: Intracellular drug release nanosystems. Mater Today 15: 436-442, 2012.

4. Li X, Kang P, Chen Z, Lal S, Zhang L, Gassensmith JJ and Qin Z: Rock the nucleus: Significantly enhanced nuclear membrane permeability and gene transfection by plasmonic nanobubble induced nanomechanical transduction. Chem Commun (Camb) 54: 2479-2482, 2018

5. Sun H, Guo B, Cheng R, Meng F, Liu H and Zhong Z: Biodegradable micelles with sheddable poly(ethylene glycol) shells for triggered intracellular release of doxorubicin. Biomaterials 30: 6358-6366, 2009.

6. Martin AL, Bernas LM, Rutt BK, Foster PJ and Gillies ER: Enhanced cell uptake of superparamagnetic iron oxide nanoparticles functionalized with dendritic guanidines. Bioconjug Chem 19: 2375-2384, 2008.

7. Komin A, Russell LM, Hristova KA and Searson PC: Peptide-based strategies for enhanced cell uptake, transcellular transport, and circulation: Mechanisms and challenges. Adv Drug Deliv Rev 110-111: 52-64. 2017.

8. Li XY, Guo SY, Zhu CL, Zhu QL, Gan Y, Rantanen J, Rahbek UL, Hovgaard L and Yang MS: Intestinal mucosa permeability following oral insulin delivery using core shell corona nanolipoparticles. Biomaterials 34: 9678-9687, 2013.

9. Mohanraj VJ, Barnes TJ and Prestidge CA: Silica nanoparticle coated liposomes: A new type of hybrid nanocapsule for proteins. Int J Pharm 392: 285-293, 2010.

10. Li X, Chen D, Le C, Zhu C, Gan Y, Hovgaard L and Yang M: Novel mucus-penetrating liposomes as a potential oral drug delivery system: Preparation, in vitro characterization and enhanced cellular uptake. Int J Nanomedicine 6: 3151-3162, 2011.

11. Jiang J, Liu S, Wang $\mathrm{C}$ and Zhang H: Overcoming multidrug resistance by on-demand int racellular release of doxorubicin and verapamil. J Nanomater 2018: 7, 2018.

12. Zhang Y, Huang L, Li Z, Ma G, Zhou Y and Han G: Illuminating cell signaling with near-infrared light-responsive nanomaterials. ACS Nano 10: 3881-3885, 2016.

13. Li N, Yu ZZ, Pan W, Han YY, Zhang TT and Tang B: A near-infrared light-triggered nanocarrier with reversible DNA valves for intracellular controlled release. Adv Funct Mater 23: 2255-2262, 2013.

14. Yavuz MS, Cheng Y, Chen J, Cobley CM, Zhang Q, Rycenga M, Xie J, Kim C, Song KH, Schwartz AG, et al: Gold nanocages covered by smart polymers for controlled release with near-infrared light. Nat Mater 8: 935-939, 2009.

15. Weissleder R: A clearer vision for in vivo imaging. Nat Biotechnol 19: 316-317, 2001.

16. Li X, Che Z, Mazhar K, Price T and Qin Z: Ultrafast near-infrared light-triggered intracellular uncaging to probe cell signaling. Adv Funct Mater 27: 1605778, 2017.

17. Bignami A, Hosley M and Dahl D: Hyaluronic acid and hyaluronic acid-binding proteins in brain extracellular matrix. Anat Embryol (Berl) 188: 419-433, 1993.

18. Surace C, Arpicco S, Dufay-Wojcicki A, Marsaud V, Bouclier C, Clay D, Cattel L, Renoir JM and Fattal E: Lipoplexes targeting the CD44 hyaluronic acid receptor for efficient transfection of breast cancer cells. Mol Pharm 6: 1062-1073, 2009.

19. Oh EJ, Park K, Kim KS, Kim J, Yang JA, Kong JH, Lee MY, Hoffman AS and Hahn SK: Target specific and long-acting delivery of protein, peptide, and nucleotide therapeutics using hyaluronic acid derivatives. J Control Release 141: 2-12, 2010. 
20. Prince ME, Sivanandan R, Kaczorowski A, Wolf GT, Kaplan MJ, Dalerba P, Weissman IL, Clarke MF and Ailles LE: Identification of a subpopulation of cells with cancer stem cell properties in head and neck squamous cell carcinoma. Proc Natl Acad Sci USA 104: 973-978, 2007.

21. Lee H, Lee K, Kim IK and Park TG: Synthesis, characterization, and in vivo diagnostic applications of hyaluronic acid immobilized gold nanoprobes. Biomaterials 29: 4709-4718, 2008.

22. Jiang M, Gan L, Zhu C, Dong Y, Liu J and Gan Y: Cationic core-shell liponanoparticles for ocular gene delivery. Biomaterials 33: 7621-7630, 2012.

23. Fomina N, Sankaranarayanan J and Almutairi A: Photochemical mechanisms of light-triggered release from nanocarriers. Adv Drug Deliv Rev 64: 1005-1020, 2012.
24. Cheng R, Feng F, Meng F, Deng C, Feijen J and Zhong Z: Glutathione-responsive nano-vehicles as a promising platform for targeted intracellular drug and gene delivery. J Control Release 152: 2-12, 2011.

25. Anderson LJ, Hansen E, Lukianova-Hleb EY, Hafner JH and Lapotko DO: Optically guided controlled release from liposomes with tunable plasmonic nanobubbles. J Control Release 144: 151-158, 2010.

26. Fuhrmann G, Serio A, Mazo M, Nair R and Stevens MM: Active loading into extracellular vesicles significantly improves the cellular uptake and photodynamic effect of porphyrins. J Control Release 205: 35-44, 2015. 